The index of refraction is a little higher than that of quartz and a little lower than that of monazite, while the double refraction is low.

These optical properties at once show that the silicate observed in the monazite is not a thorium silicate or thorite, but is probably feldspar.

The microscopic investigation therefore bears out the chemical investigation that thorium is not present in monazite in the form of thorium silicate.

Qtintitative Laboratory, April, Igog.

\title{
AN AUTOMATIC PIPETTE FOR CAUSTIC SODA SOLUTION.
}

By Francis G. BENEdTCT,

Received March 30 , 1909

With the introduction of the rapid and extremely accurate Kjeldahl method for the determination of nitrogen, this estimation has become more or less of a routine in a large number of laboratories where the determinations are carried out with great rapidity and in great numbers. A large number of improvements have been suggested in the original method as outlined by Kjeldahl, all of which are designed to improve accuracy of determination and facility of manipulation. While the use of catalyzing agents, special stills and automatic pipettes have all met with great success, the attempts to facilitate the handling of rather large amounts of saturated sodium hydroxide solutions have so far met with but indifferent stccess. The most successful apparatus is the siphon of Durig. ${ }^{1}$

In connection with the establishment of a special room for Kjeldahl analysis in the Nutrition Laboratory in Boston, it seemed desirable to introduce some appliance for making the handling of this reagent more automatic and less troublesome than is commonly the case. The apparatus described herewith has been perfected and is now in constant use in the laboratory, giving excellent results.

The apparatus uses the method of displacement in that a given volume of the liquid is displaced by a plunger (E) descending into the liquid and allowing the desired quantity to overflow. As the plunger descends, it automatically shuts off the feed from the reservoir (D) so that the exact amount of liquid desired is displaced and no more. In such an apparatus two points must be taken into consideration: First, the great viscosity of the solution, calling for large caliber glass tubes; second, the impossibility of securing a valve to close off the supply constructed of any material other than rubber. The use of rubber tubing for an apparatus of this kind is open to serious objections, but with the arrangement of the pinch-cock, as shown in the diagram, it can be seen that that portion of the rubber subjected to most wear, i. e., the part (B)

i Biochem. Z., 4, 72 (1907). 
which is compressed for closure, can be readily replaced without disturbing the whole apparatus. Furthermore, the $\mathrm{V}$-shaped piece of rubber (A) minimizes the wear on (B).

The caustic soda solution is placed in a large carboy on a shelf above the valve. The apparatus as in use in the laboratory is constructed of simple materials but can obviously be made in large part of glass. We have used an ordinary Welsbach lamp chimney (D) with a 2-hole rubber stopper (G) inserted in the base. In one of the holes is inserted a glass tube leading from the carboy of caustic soda solution and in the other a doubly bent glass tube (F) which serves as an overflow for the expelled liquid. In the top of the chimney is placed a rubber stopper with one hole in it, carrying a small glass or brass tube which acts as a guide for an iron rod (C) fastened to the top of a small glass bottle (E) which fits loosely the interior diameter of the glass chimney. By means of this iron rod the small bottle inside can be raised or lowered, and to add weight to the bottle it is filled with shot. A pulley running from the shelf overhead allows the weighted bottle to be lifted. As the bottle is lifted, the shut-off from the reservoir is simultaneously opened, allowing the caustic soda to flow into the lamp chimney. This weighted bottle is held suspended until the level of the reagent in the glass reservoir rises to a mark which can be arbitrarily adjusted. At that moment the weight is released, it descends into the liquid, simultaneously shuts off the supply and displaces a definite amount of caustic soda solution which flows

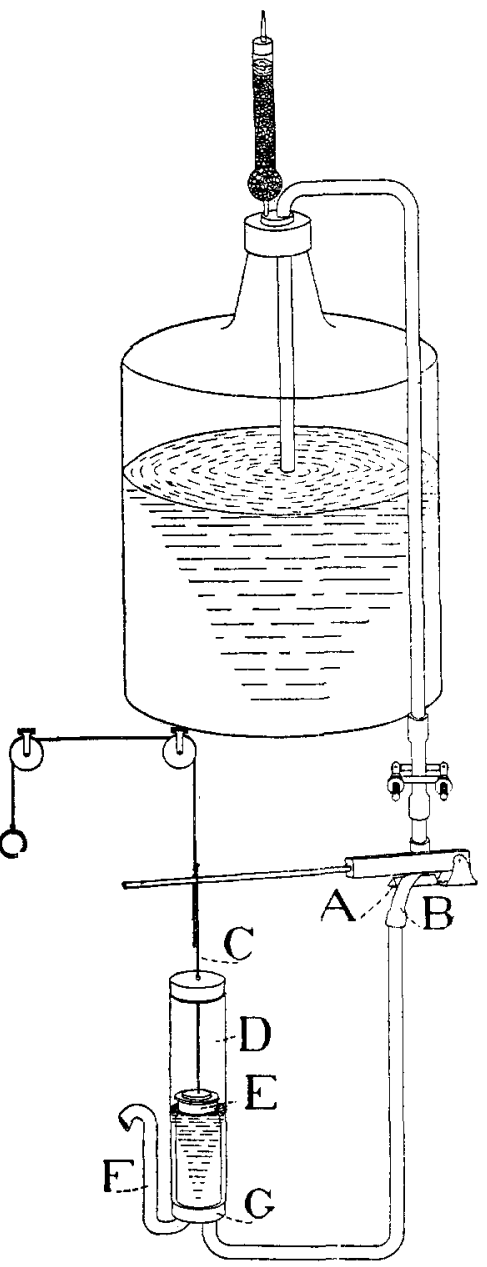
out through the overflow. The neck of the Kjeldahl flask can be held beneath the overflow and the alkali delivered into the flask with great regularity and satisfaction. For purposes of regular use, the solution can be made any strength desired and the amount delivered adjusted by an arbitrary scale on the lamp chimney until such an amount of alkali is delivered as will neutralize wholly all the sulphuric acid renaining 
in the Kjeldahl flask after digestion. In practice, the adjustment is so made that the apparatus delivers each time about 80 cubic centimeters of strong sodium hydroxide solution.

NUTRITIOX I,ABORATORY,

CARNEGIE INSTITETION OF WASHINGTON,

VILA STRLET, BOSTON, MASS.

\section{NOTE.}

Glass-cutting by Mcans of an Elcctric Wire..... While it is of ten necessary, in laboratory practice, to cut bottles and other glassware for the construction of special apparatus, the methods commonly used are somewhat tedious, besides requiring considerable manipulative skill for good results. The following simple device, however, will be found to combine rapidity with precision, even in the hands of an inexperienced operator. For some time the writer has been using it successfully, and, not knowing of its ever having been used before, publishes it with the idea that it might possibly be of interest to other experimenters.

The method consists in passing a current through a thin resistancewire which has previously been wound about the glass vessel and carefully adjusted. For this purpose the rro-volt lighting circuit may be used, and, aside from the resistance-wire just mentioned, the only apparatus required is a couple of pieces of No. I 8 insulated copper wire, a pair of ringstands or other heavy objects, and a suitable rheostat. 'The vessel must be perfectly dry, and should have a scratch about I/4 inch long made upon its surface, either on the emery-wheel or with a file, in order to provide a starting point for the crack. After joining the ends of the resistance-wire to the copper leads, fixing the latter to the ringstands at the desired height, and connecting the rheostat in series, the resistance-wire is fastened in a single loop around the glass vessel, so as to pass directly over the file-mark, and drawn taut by means of the ringstands. A particle of asbestos paper is then inserted between the crossed ends of the loop, to prevent contact, and enough current is turned on to heat the wire to dull redness. In a few seconds a crack forms at the file-mark and spreads rapidly around the vessel-frequently the crack starts and snaps across all at once; in either case, it will be found to have followed the path of the resistance-wire perfectly, so that the trueness of the fracture depends entirely on the smoothness and alignment of the wire.

Apart from the illustration just given, which merely describes the cutting of a bottle in two, the method may equally well be applied to more intricate cases, where the fracture is to be a curve or a spiral. 